\title{
Vicode: 3d Barcode with Embedded Video Using Histogram Shifting Based Reversible Data Hiding
}

\author{
Haritha $\mathbf{H}^{1}$, Anish Kumar $\mathbf{B}^{2}$ \\ ${ }^{1}$ MEA Engineering College, State Highway 39, Nellikunnu-Vengoor, Perinthalmanna, Malappuram, Kerala
}

${ }^{2}$ Assistant Professor, MEA Engineering College, State Highway 39, Nellikunnu-Vengoor, Perinthalmanna, Malappuram, Kerala

\begin{abstract}
QR codes are widely used as an interface to connect advertisement contents and potential customers. The appearance of a traditional $2 D$ barcode pattern is too obtrusive for integrating into an aesthetically designed advertisement, no visual hint about the encoded information is provided before the barcode is successfully decoded. These two issues limit the potential customers interest in scanning the barcode and reduce the chance of successful customer engagement. This paper proposes a new picture embedding $2 D$ barcode, called PiCode, which mitigates these two limitations. This motivates to increase barcode storage capacity by developing video barcode, a way to integrate a video clip into a series of $2 \mathrm{D}$ barcodes (ViCode) is developed. To realize ViCode, new modulation and demodulation schemes are designed. This paper also gives an overview of different steganographic techniques such as histogram Shifting based Reversible Data Hiding Scheme, Reversible Data Embedding Using a Difference Expansion, An LSB Data Hiding and compare these methods according to PSNR, MSE and SSIM. ViCode has been implemented in Matlab on a PC.
\end{abstract}

Keywords: 3D Barcode, Embedded Video, Encoder, Decoder, Perceptual Quality, Decoding Robustness

\section{Introduction}

2D Barcodes are rapidly emerged as a widely used marketing, health care applications, industrial applications, retail applications, manufacturing, and product tracking etc. Advertisers are taking advantage of barcodes by using them to reach out to customers in a more interactive and interesting way. This invention aims at developing ViCode, a human readable 3D barcode technology, distinguished from common binary 2D barcodes which do not convey meaning-ful visual information to customers[3]. 3D barcodes increases barcode storage capacity, which roughly refers to a video series of $2 \mathrm{D}$ barcodes, and to embed in it a low resolution video clip, called ViCode. ViCode system mainly emphasis on the new kind encoding and decoding algorithms. This paper explains various steganographic techniques, traditional 2D barcodes and PiCode(Picture embedding 2D barcode). Histogram Shifting based Reversible Data Hiding Scheme, Reversible Data Embedding Using a Difference Expansion and An LSB Data Hiding are the various data embedding techniques and compare these methods according to PSNR, MSE and SSIM. In LSB method the embedding is done by replacing the least significant bit position of image pixels with the bits of secret data. Change occur at only one bit so intensity of image is not effected too much. In histogram based data hiding technique the crucial information is embedded into the image histogram. The peak point and zero point are identified for pixel shifting. In Difference Expansion(DE) calculate the differences of neighboring pixel values, and select some difference values. The original data will all be embedded into the difference values.

In this work, introduced a histogram shifting based reversible data hiding for data embedding. Histogram shifting based method have high payload performance and visual quality greater than $33 \mathrm{~dB}$. ViCode provide eye appealing visual information, such as a picture or video clip, meaningful to people. Compare previous methods with the proposed method and analyse the perceptual quality.

\section{Literature Survey}

\section{Steganographic Techniques}

Spatial domain techniques are :

1) Least significant bit (LSB) Difference

2) Expansion (DE)

3) Histogram Shifting

\subsection{Least Significant Method(LSB)}

Least significant bit (LSB) insertion is a simple approach to embedding information in a cover imag in least significant bit position[14]. Intensity of image is only change by ' 0 ' or ' 1 ' after hiding the information. Change occur at only one bit so intensity of image is not effected too much[4].

\subsection{Difference Expansion Method(DE)}

Difference expansion (DE) is one of the most important techniques which are used for reversible data hiding. This technique have high efficiency and simplicity.

In DE calculate the differences of neighboring pixel values, and select some difference values. The original data will all be embedded into the difference values[12].

\subsection{Histogram Shifting Based Method}

Histogram based data hiding is another commonly used data hiding method. Histogram shifting technique prevents overflow and underflow problems. The pixel histogram is generated by counting the frequency of pixel values from 0 to 255 in the image. Then the peak point and zero point are identified for pixel shifting. If a pixel is between the peak point and zero point, the pixel is shifted by one from the peak point towards the zero point. Then, the secret data is 
embedded into the pixel belonging to the peak point. The embedding rule is that: if the secret bit equals to ' 1 ' and the pixel belongs to the peak point, then the pixel value is increased by one; else if the secret bit equals to ' 0 ' and the pixel belongs to the peak point, then the pixel value remains unchanged. In the data extraction phase, the secret bit ' 1 ' is unchanged. In the data extraction phase, the secret bit ' 1 ' is extracted if the pixel belongs to the peak point plus one. The secret bit ' 0 ' is extracted if the pixel belongs to the peak point. After all secret bits have been extracted, the image can be fully restored by shifting the pixels back to between the peak point and zero point[13].

\section{Mean Squared Error (MSE)}

Measure of the quality of an estimator is called as Mean Squared Error is shown in equation (1)

$$
\mathrm{MSE}=\frac{1}{m n} \sum_{i=0}^{m-1} \sum_{j=0}^{n-1}[I(i, j)-K(i, j)]^{2}
$$

In equation (1) $\mathrm{m}, \mathrm{n}$ are the number of rows and columns in the cover image matrix, $\mathrm{K}(\mathrm{i}, \mathrm{j})$ is the pixel value from stego image and $I(i, j)$ is the pixel value from cover image. Higher value of MSE indicates dissimilarity of between compared images.

\section{Peak Signal to Noise Ratio (PSNR)}

PSNR is the error metric used to compare image compression quality and it is the ratio between the maximum possible power of a signal and the power of corrupting noise is shown in equation (2). This ratio is used as a quality measurement between the original and a compressed image. PSNR measures in decibels.

$$
\text { PSNR }=20 \log _{10}\left(M A X_{I}\right)-10 \log _{10}(M S E)
$$

\section{Structural Similarity (SSIM)}

SSIM is defined as measuring the similarity between two images. The SSIM index is calculated as in equation (3).

$$
\operatorname{SSIM}(\mathrm{x}, \mathrm{y})=\frac{\left(2 \mu_{x} \mu_{y}+c_{1}\right)\left(2 \sigma_{x y}+c_{2}\right)}{\left(\mu_{x}{ }^{2}+\mu_{y}{ }^{2}+c_{1}\right)\left(\sigma_{x}{ }^{2}+\sigma_{y}{ }^{2}+c_{2}\right)}
$$

$\mu_{x}$ is the average of $\mathrm{x}$

$\mu_{y}$ is the average of $\mathrm{y}$

$\sigma_{x}{ }^{2}$ is the variance of $\mathrm{x}$

$\sigma_{y}{ }^{2}$ is the variance of $\mathrm{y}$

$\sigma_{x y}$ is the variance of $\mathrm{x}$ and $\mathrm{y}$

$C_{1}$ and $C_{2}$ are stabilize the division with weak denominator $\mathrm{L}$ the dynamic range of pixel values.

\section{Performance Comparison}

Table 1: Performance comparison of various steganographic techniques

\begin{tabular}{|c|l|c|c|c|}
\hline Parameters & LSB & $\begin{array}{c}\text { Difference } \\
\text { Expansion }\end{array}$ & $\begin{array}{c}\text { Intensity } \\
\text { Modulation }\end{array}$ & $\begin{array}{c}\text { Histogram } \\
\text { Based Shifting }\end{array}$ \\
\hline PSNR & Low & Low & High & High \\
\hline MSE & High & High & Low & Low \\
\hline SSIM & Low & Low & High & High \\
\hline
\end{tabular}

From the Table 1 it can be find that Histogram shifting based method is more better than that of other techniques. Least significant based method have low peak signal to noise ratio, high mean squared error value and low structural similarity. Histogram based method have high peak signal to noise ratio, low mean squared error value and high structural similarity. Difference expansion method have low peak signal to noise ratio, high mean squared error value and low structural similarity.

Least significant method exhibits high perceptual transparency and low degradation in the image quality. The main disadvantage of least significant bit method is low robustness to malicious attacks. Difference expansion exhibits high visual quality and high computational complexity. The main disadvantage of difference expansion is vulnerable to accident. Histogram shifting method have high payload performance and visual quality greater than $33 \mathrm{~dB}$.

\section{Review of beautified $Q R$ code}

Image embedding in $\mathrm{QR}$ code using half toning Technique: $\mathrm{QR}$ code values are transformed in to luminance values of image which is to be embedded in such way that luminance level is increased for light region of code and decreased for dark region of code [6].

Image embedding in QR code using multi resolution halftoning technique: This method differs than other in case of using multi resolution half toning instead of half toning for generation of masks [7].

Image embedding in QR code using genetic algorithm: In this technique only difference is using a genetic algorithm at optimization level. This reduces processing time required for the embedding process [8].

Image embedding in QR code using DW: Input to the system is color image and color QR code. At next stage image is divided in to low frequency and high frequency information. Both low frequency and high frequency information decomposed in to low frequency area and high frequency area that is LL, LH, HL and HH[10]. In it edge is detected using DWT. Then mask is generated with high frequency extraction further it is optimized and integrated to give final embedded image. Quality and visual appearance of final result is improved.

\section{Methodology}

Two dimensional barcodes have been widely used as a bridge to connect potential customers and advertisement contents. The appearance of a traditional 2D barcode pattern is often too obtrusive which are not aesthetically good. No human readable information is provided before a successful decoding is accomplished. This paper proposes a new picture embedding 2D barcode, called PiCode, which mitigates these two limitations. By extending the idea, a way to integrate a video clip into a series of 2D barcodes (called ViCode) is also developed. ViCode is shown in figure 2. PiCode, ViCode has been implemented in Matlab on a PC. 
International Journal of Science and Research (IJSR)

ISSN (Online): 2319-7064

Index Copernicus Value (2015): 78.96 | Impact Factor (2015): 6.391

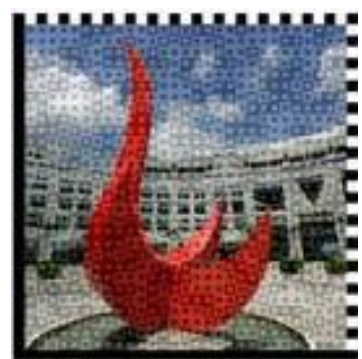

Figure 1: PiCode

The proposed PiCode system is includes both encoding and decoding algorithms. In the encoding part, the modulation scheme will be presented to illustrate how PiCode preserves the perceptual quality of the embedded image while minimizing the interference of the latter incurred on the modulation waveform. The algorithms for performing corner detection, module alignment and demodulation will be described in the decoding part. Two dimensional (2D) barcodes are widely used as a bridge to link the potential customers and advertisement contents[11]. The appearance of a traditional 2D barcode pattern is too obtrusive, which are not aesthetically good and no visual hint about the encoded information content is provided before the barcode is successfully decoded. This paper has designed a novel picturesque 2D barcode, called PiCode as shown figure 1 . Picode and ViCode achieves best perceptual quality of the embedded image and the decoding robustness of the encoded message when compairing with existing beautified QR codes. The modulation waveform is constructed by setting the intensity of an image block to two levels, dark or bright, to represent bit 0 and 1, respectively. PiCode is designed with fixed patterns to avoid distortions on the embedded image. PiCode Encoding PiCode system consist both encoding and decoding algorithms. PiCode encoding process can be divided into input processing and the PiCode generation. Input processing method contains, Source Coding and Channel Coding.

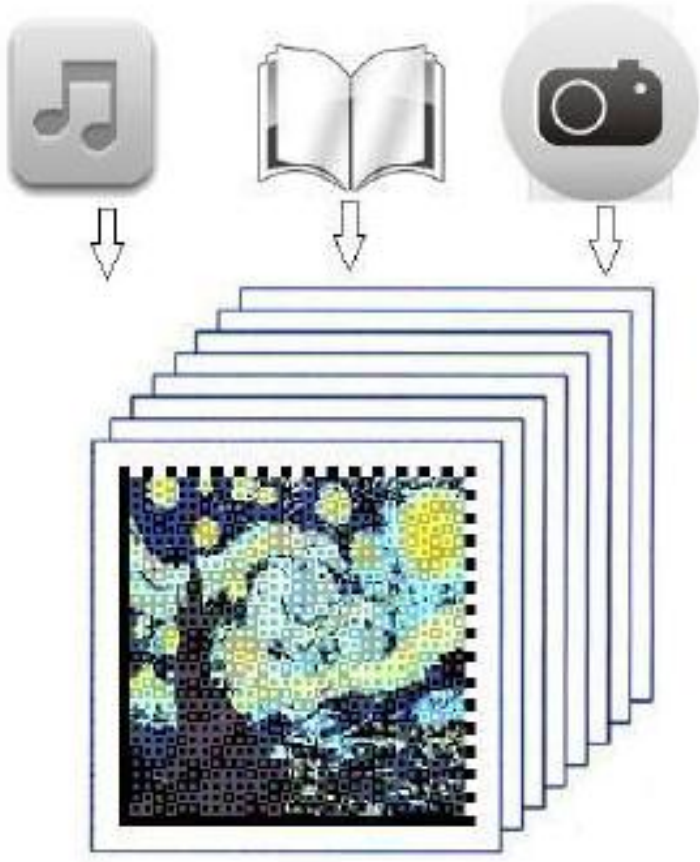

Figure 2: ViCode

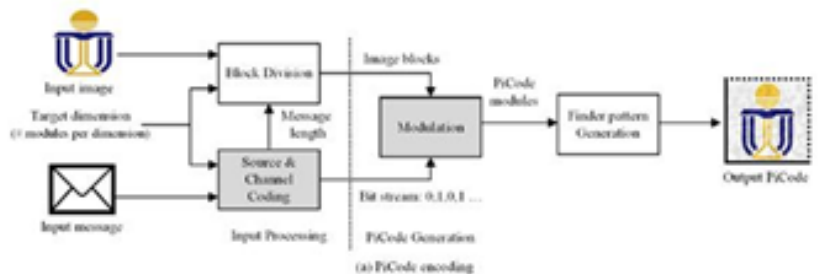

Figure 3: PiCode Encoding

\subsection{PiCode Encoding}

In source coding code data to more efficiently represent the information and in channel Coding Code data for transmission over a noisy communication channel. First the input message is converted into a bit stream through source coding and channel coding to improve the efficiency and robustness of the encoded message[1]. The input image is divided into 2D grid of image blocks according to the user's input. Each block consists of kxk pixels. PiCode generation part image blocks of $\mathrm{k} * \mathrm{k}$ pixels are modified using adaptive modulation scheme. The modulation waveform is constructed by setting the intensity of an image block to two levels, dark or bright, to represent bit 0 and 1 , respectively. Then, a layer of finder pattern is added to the exterior of the modulated 2D grid of image blocks to form the PiCode.

Figure 3 shows picode encoding.

Channel coding and the modulation scheme are essential in balancing the decoding robustness and perceptual quality. channel coding is to protect the information bits against errors after the demodulation step. channel coding is to protect the information bits against errors after the demodulation step. In PiCode, a Reed-Solomon (RS) code over the finite field galois field is adopted.

The code rate is adaptive to the message length . The shorter the input message, the smaller the code rate and vice versa. This is designed to maximize the error correction capability under a given pattern size and message length . To ensure decoding robustness, the maximum code rate is set as 5/6 which ensures that the code is capable of correcting one erroneous byte out of every 12 bytes. In modulation scheme, the aim is to represent a message, e.g., bit 0 or 1 , by varying some physical parameter (e.g. intensity) of the modulation

waveform. The modulation scheme considering both decoding robustness and perceptual quality. For the color components of the embedded image, they should be modified with the least perceptual differences when compared with the original colors . Modulation operation in the YUV color space which treats the chrominance and luminance components independently. During color rendering, the two chrominance components, $\mathrm{U}$ and V, should be kept the same as the original ones so that the perceptual difference between the original and modulated colors are minimized, while the luminance component $(\mathrm{Y})$ is modified according to the proposed modulation scheme.

\subsection{PiCode Decoding}

In PiCode decoding part the captured PiCode image is converted to grayscale then the grayscale image is binarized. The potential barcode regions are checked against the 


\section{International Journal of Science and Research (IJSR) \\ ISSN (Online): 2319-7064 \\ Index Copernicus Value (2015): 78.96 | Impact Factor (2015): 6.391}

detection criterion. If the check is happen, the four corners are acquired, otherwise the image will be discarded and the decoding process will be reinitiated with another image frame. The perspective distortion is then estimated based on the barcode corner locations, and compensated on the graylevel image. In the module alignment, the region for each PiCode module is obtained based on broken line parts of the finder patterns. The demodulation process is the reverse of the modulation process by inspecting the intensity differences between the inner and outer parts of each module. The modulated bit in each module is restored by the demodulation operation. Then message is obtained by applying channel and source decoding to the demodulated bits. corner detection, module alignment and demodulation included in the decoding part. Figure 4 shows picode decoding.

Coarse-Fine Corner Detection: The corner detection algorithm locates four extreme corners of the barcode from the captured image [2].

Module Alignment: The module alignment step slices the barcode region into image blocks with reference to the black and white alternations. Each block corresponds to one module which is then input to the succeeding demodulation step. The accuracy of the module alignment step is therefore critical to the decoding performance.

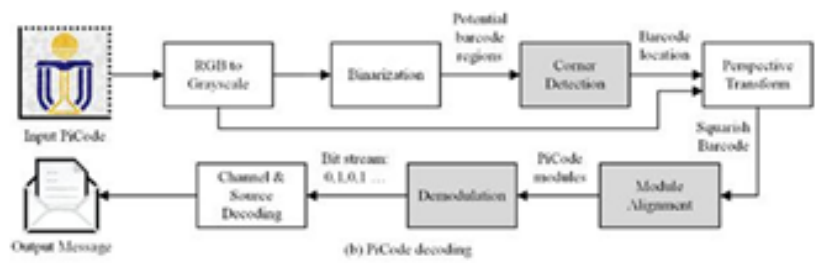

Figure 4: PiCode Decoding

Demodulation: In the demodulation step, each received module resulted from the module alignment step is analyzed to retrieve the data bit. The demodulation scheme is designed according to the modulation scheme described. Before the demodulation operation, each module is first resampled with the bilinear interpolation into $8 \times 8$ pixels since it is the minimum size required for our demodulation algorithm, luminance change, e.g., a sharp edge and a corner.

To demonstrate the practicality of the proposed PiCode, implemented algorithm in PC. With an unoptimized Matlab implementation on a PC with Intel i5 CPU and 8 GB ram, a PiCode with embedded image of size $512 \times 512$ pixels can be encoded within 1 second. It costs $\sim 1.4$ seconds to decode a low capacity PiCode image captured with a resolution of $720 \times 720$ pixels, while the decoding time takes $\sim 2.1$ seconds for the high capacity version[9].

In the future, the unobtrusive pilot symbols will be embedded into the PiCode center to serve as center alignment pattern and training symbols for the camera response function. To evaluate distortions, multiscale structural similarity (MS-SSIM) metric is employed. The metric ranges from 0 to 1 . A higher score indicates higher similarity between the original and processed images, and therefore less distortions are incurred by the picture embedding process.
By extending the idea, a way to integrate a video clip into a series of 2D barcodes (called ViCode) is also developed[5]. ViCode can be viewed as a 3D barcode extension of PiCode. As a 3D barcode, ViCode can store much more informationthan any single 2D barcode because of the addedtime dimension. In addition, ViCode can also show an embedded video clip or animation, which is ideal for attracting consumers eyeballs in mobile marketing applications. The ViCode system produces, instead of a single barcode image, a series of $2 \mathrm{D}$ barcodes, which can be shown as a video clip or even a video stream. The camera then captures each frame of ViCode and decode these frames in combination, which allows a much higher data capacity than decoding frame by frame. To achieve good performance, the interframe and intraframe channel coding should be optimized.

\section{Experimental Results}

Bit error rate (BER) is the number of bit errors per unit time.

Table 2: Performance Summary of Different Barcodes.

\begin{tabular}{|c|c|c|c|c|}
\hline Metric & Halftone QR & QR Image & PiCode & ViCode \\
\hline Average BEP & 0.008 & 0.021 & 0.039 & 0.039 \\
\hline
\end{tabular}

The 3D barcode with embedded video serves both the human visual system and the barcode decoder. Therefore, experiments have been conducted to study both the perceptual quality and the decoding robustness of ViCode as well as existing beautified QR codes. The perceptual quality contributes to the visual attractiveness of the barcode, while the decoding robustness affects the user experience during the decoding process. Both are critical for the targeted advertisement applications. Table 2 shows Performance Summary of Different Barcodes based on Bit Error Probability (BEP).

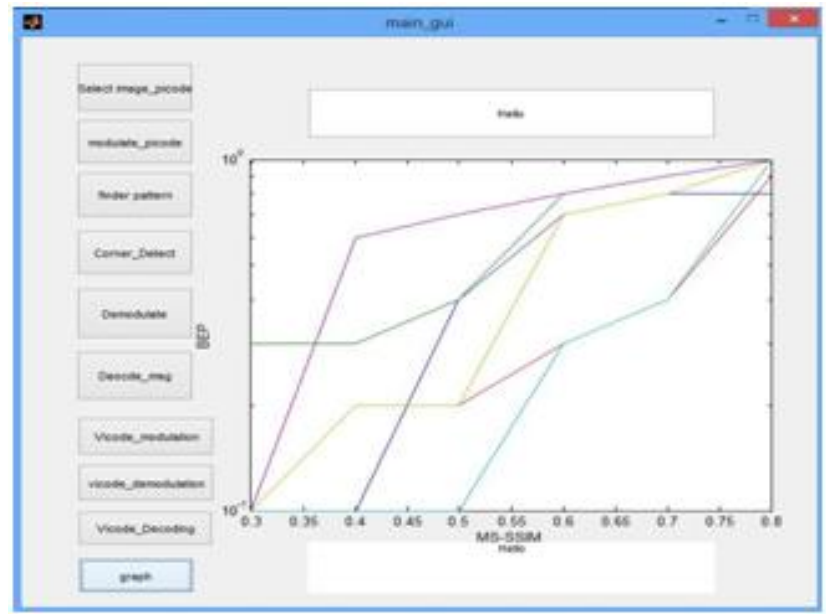

Figure 5: Effect of varying the embedding intensity on perceptual quality and decoding robustness.

The multiscale structural similarity (MS SSIM) metric is employed in our experiment to cater for different viewing conditions, such as different resolutions and physical sizes, which are important for barcodes with different capacities[15]. The metric ranges from 0 to 1 . A higher score indicates higher similarity between the original and processed images, and therefore less distortions are incurred by the picture embedding process. Bit error probability

Volume 6 Issue 7, July 2017 www.ijsr.net 


\section{International Journal of Science and Research (IJSR) \\ ISSN (Online): 2319-7064 \\ Index Copernicus Value (2015): 78.96 | Impact Factor (2015): 6.391}

(BEP) is used to measure the decoding performance. BEP is the fraction of the erroneous bits after the demodulation step. Figure 5 shows Effect of varying the embedding intensity on perceptual quality and decoding robustness. Halftone QR code and QR Image have more favorable BEP performance under both low and high capacity conditions.

\section{Conclusion}

Both our PiCode and ViCode can offer excellent performance in the terms of decoding robustness and data capacity, compared with existing 2D and 3D barcodes available in mobile phone platforms. More importantly, they can also provide eye-appealing visual information, such as a picture or video clip, meaningful to people. They are therefore very promising tools for mobile marketing applications. By integrating PiCode or ViCode into a company logo, commodity packages, electronic billboards, digital signage, TV advertisement, and so on, it can effectively connect potential customers to commercial products. In the near future, when Google glasses become popular, PiCode and ViCode may appear everywhere because they can deliver various form of data to people at no (or very low) cost while they are simply looking at a PiCode or ViCode in the guise of an Eye appealing picture or video clip.

\section{References}

[1] Chen, C., Huang, W., Zhou, B., Liu, C., and Mow, W. H. (2016). Picode: A new picture-embedding 2d barcode. IEEE Transactions on Image Processing, 25(8):3444-3458.

[2] Chen, C. and Mow, W. H. (2014). Poster: A coarse-fine corner detection approach for two-dimensional barcode decoding. In Proceedings of the 20th annual international conference on Mobile computing and networking, pages 351-354. ACM.

[3] Gao, J. Z., Prakash, L., and Jagatesan, R.(2007). Understanding 2d-barcode technology and applications in m-commerce-design and implementation of a $2 \mathrm{~d}$ barcode processing solution. In Computer Software and Applications Conference, 2007. COMPSAC 2007. 31st Annual International, volume 2, pages 49-56. IEEE.

[4] Gupta, S., Goyal, A., and Bhushan, B.(2012). Information hiding using least significant bit steganography and cryptography. International Journal of Modern Education and Computer Science, 4(6):27.

[5] Huang, W. and Mow, W. H.(2013). Picode: 2d barcode with embedded picture and vicode: $3 \mathrm{~d}$ barcode with embedded video. In Proceedings of the 19th annual international conference on Mobile computing \& networking, pages 139-142. ACM.

[6] Kato, H. and Tan, K. T. (2007). Pervasive 2d barcodes for camera phone applications IEEE Pervasive Computing, 6(4).

[7] Lin, S.-S., Hu, M.-C., Lee, C.-H., and Lee, T.-Y. (2015). Efficient qr code beautification with high quality visual content IEEE Transactions on Multimedia , 17(9):15151524.

[8] McCune, J. M., Perrig, A., and Reiter, M. K. (2005). Seeing-is-believing: Using camera phones for humanverifiable authentication. In Security and privacy, 2005 IEEE symposium on, pages 110-124. IEEE.
[9] Mow, W. H., Au, C. Y., Chiu, C. Y., Li, K. S., and Huang, W. (2015). Embedding visual information in a two-dimensional bar code. US Patent 8,948,445.

[10] Muammar, H. and Dragotti, P. L. (2013). An investigation into aliasing in images recaptured from an lcd monitor using a digital camera. In Acoustics, Speech and Signal Processing (ICASSP), 2013 IEEE International Conference on , pages 2242-2246. IEEE.

[11] Ohbuchi, E., Hanaizumi, H., and Hock, L. A. (2004). Barcode readers using the camera device in mobile phones. In Cyberworlds, 2004 International Conference on , pages 260-265. IEEE.

[12] Tian, J. (2003). Reversible data embedding using a difference expansion. IEEE transactions on circuits and systems for video technology, 13(8):890-896.

[13] Tseng, C.-C., Chiu, Y.-H., and Chou, Y.-C. (2015). A histogram shifting-based reversible data hiding scheme using multi-pattern strategy. In Intelligent Information Hiding and Multimedia Signal Processing (IIH-MSP), 2015 International Conference on, pages 125-128. IEEE.

[14] Tyagi, V.(2012). Image steganography using least significant bit with cryptography. Journal of global research in computer science, 3(3):53-55.

[15] Wang, Z., Simoncelli, E. P., and Bovik, A. C.(2003). Multiscale structural similarity for image quality assessment. In Signals, Systems and Computers, 2004. Conference Record of the Thirty-Seventh Asilomar Conference on, volume 2, pages 1398-1402. IEEE.

\section{Author Profile}

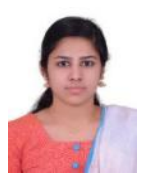

Haritha $\mathbf{H}$ received her B.Tech. degree in computer science and engineering from the MEA Engineering College, Kerala, in 2015. Right now she is pursuing her M. Tech degree in computer science at MEA Engineering College, Kerala from 2015 to 2017. Her research interests lie in Image Processing.

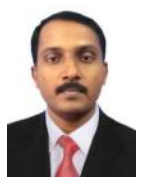

Anish Kumar B received his B.Tech. degree in computer science and engineering from the college of Engineering, Karunagappally, Kerala, in 2006. He had completed his M. Tech degree in computer science at college of Engineering, Chengannur, Kerala from 2014. His research interests lie in Image Processing. 